\title{
Consolidation des connaissances nationales sur le trait de côte et son évolution
}

\section{François HÉdOU ${ }^{1}$, Boris LECLERC ${ }^{1}$, Yann DENIAUD ${ }^{1}$, Stéphane COSTA ${ }^{2}$}

1. Cerema, direction Eau, mer et fleuves, 155, rue Pierre Bouguer, BP5, 29280 Plouzané, France.

francois.hedou@cerema.fr ; boris.leclerc@cerema.fr ; yann.deniaud@cerema.fr

2. Normandie Univ, Unicaen, CNRS, LETG-Caen, UMR-CNRS 6554, Esplanade de la Paix, 14032 Caen cedex, France.

stephane.costa@unicaen.fr

\section{Résumé :}

L'indicateur national de l'érosion côtière, réalisé dans le cadre de la Stratégie nationale de gestion intégrée du trait de côte (SNGITC), fournit des taux d'évolution historique du trait de côte selon des profils disposés tous les 200 mètres le long du littoral français. Afin de disposer d'informations sur des linéaires côtiers en évolution et non plus des chiffrages en nombre de profils, peu adaptés à un large public, les données ponctuelles de l'indicateur national sont reportées sur un référentiel connu et partagé, à savoir la limite continue Histolitt. Cette dernière est également découpée selon la géomorphologie du trait de côte et est complétée avec les informations apportées par la cartographie nationale des ouvrages et aménagements côtiers. En croisant ces différentes sources de données, il devient possible d'identifier les secteurs du trait de côte connaissant une problématique liée à l'érosion côtière et à la submersion marine. Il s'agit des secteurs présentant un recul observé du trait de côte, mais aussi ceux ayant nécessité la mise en place d'ouvrages spécifiques de lutte contre l'érosion marine (épis et brise-lames) et la construction d'ouvrages se substituant au trait de côte naturel (digues, murs et perrés). Ainsi, à l'échelle nationale (métropole et DROM, hors Guyane, et hors ports et estuaires), $1800 \mathrm{~km}$ de trait de côte au moins (selon le tracé de Histolitt) connaissent une problématique liée à l'érosion côtière et à la submersion marine. Ils représentent 25,5\% du linéaire analysé. Cette étude montre également que $49 \%$ du linéaire des côtes basses sableuses françaises sont dans cette situation.

\section{Mots-clés :}

Trait de côte, Recul, Linéaire, Evolution, Littoral, Indicateur national, Ouvrages littoraux, Histolitt, Etude nationale, Erosion, Submersion marine. 


\section{Thème 7 - Risques côtiers}

\section{Contexte et problématique}

Afin de disposer d'un état des lieux de l'évolution du trait de côte sur l'ensemble du littoral français, la Stratégie nationale de gestion intégrée du trait de côte (SNGITC) (MEDTL, 2012) a préconisé la réalisation d'un indicateur national de l'érosion côtière. Cet indicateur national est inscrit dans la loi "Biodiversité" du 8 août 2016, par l'article L321-13 du Code de l'environnement. Produit par le Cerema, à la demande du ministère chargé de l'environnement, pour les littoraux de métropole et des départements et régions d'outre-mer (DROM), l'indicateur national de l'érosion côtière (CEREMA \& MTES, 2018) quantifie les tendances historiques de l'évolution du trait de côte (recul, stabilité ou avancée). Ces tendances sont calculées en comparant la position du trait de côte sur 2 photographies aériennes dont les dates de prise de vue sont éloignées de plusieurs décennies. Dans le détail, le calcul des taux d'évolution a été mené au droit de profils disposés tous les 200 mètres le long du littoral. Les analyses de l'indicateur ont ainsi été réalisées uniquement sur la base du nombre de profils en évolution et fournissent principalement des résultats sous la forme de pourcentages globaux. Bien que difficile à établir, la quantification des linéaires de trait de côte concernés par un recul est cependant un élément éclairant qui permet une meilleure sensibilisation à l'importance du phénomène de mobilité du trait de côte en France. Cette analyse en linéaire côtier se combine aisément aux données apportées par la cartographie nationale des ouvrages et aménagements littoraux (CEREMA \& MTES, 2017). L'objet de cette publication est ainsi de décrire le transfert des informations provenant des profils vers un trait de côte de référence et ainsi d'analyser la mobilité du trait de côte français selon une quantification linéaire.

\section{Principaux résultats de l'indicateur national selon l'analyse des profils}

La méthode mise en œuvre dans le cadre de l'indicateur national de l'érosion côtière a permis de calculer l'évolution du trait de côte sur près de 23000 profils (métropole et DROM) lorsque 2 marqueurs de position du trait de côte de même type recoupaient le même profil. En excluant les observations effectuées sur le littoral Guyanais, où la migration périodique d'importants bancs de sédiments vaseux provenant de l'Amazone rend délicate toute interprétation des résultats, l'analyse de l'évolution du trait de côte par classe de valeurs sur le territoire national montre que sur les 21683 profils où l'évolution est mesurée, $18,6 \%$ sont en recul, $11,7 \%$ sont en avancée et une large majorité $(69,7 \%)$ présentent une évolution qualifiée de "non perceptible" dans un intervalle $\pm 0,1 \mathrm{~m} / \mathrm{an}$ (HÉDOU et al., 2018).

\section{Transfert des informations provenant des profils vers un linéaire côtier}

Le transfert des informations provenant des profils vers un linéaire côtier est une opération complexe qui nécessite le choix d'un trait de côte de référence, une emprise commune aux sources de données et des traitements géomatiques. 


\section{XVI èmes Journées Nationales Génie Côtier - Génie Civil \\ Le Havre 2020}

\subsection{Tracé du trait de côte pris comme référence}

Le trait de côte pris en référence dans cette étude est le tracé Histolitt (V2), produit par le Shom et l'Ifremer (IGN \& SHOM, 2009). Celui-ci, représente la laisse des plus hautes mers astronomiques de coefficient 120 , avec des conditions météorologiques normales. Bien qu'un peu ancien (production avec les données disponibles entre 2006 et 2007), il présente l'avantage de fournir un tracé continue et homogène sur la majorité des littoraux français. Long de près de $17000 \mathrm{~km}$ en métropole et dans les DROM, le tracé Histolitt remonte très profondément dans les estuaires.

\subsection{Emprise de l'étude}

L'emprise du tracé Histolitt dépasse largement le secteur d'étude de l'indicateur national correspondant aux seuls secteurs littoraux directement exposés à l'action de la houle. L'identification des emprises communes aux 2 sources de données est donc un préalable indispensable aux processus d'affectation des données d'évolution sur le tracé Histolitt. Cela oblige indirectement à identifier sur le tracé Histolitt les zones d'estuaires et les zones portuaires non étudiées par l'indicateur national. Pour cela les données du projet Européen "Eurosion" (CE, 2004), comprenant notamment la nature du trait de côte (géomorphologie et géologie) sont exploitées : Dans le cadre du projet "Dynamiques et évolutions du littoral", ces données ont été transférées sur le tracé Histolitt et permettent d'écarter les sections situées dans les estuaires et dans les ports. D'autres ajustements sont également nécessaires pour identifier et écarter également les quelques autres zones non traitées par l'indicateur national, en particulier les îles non habitées et la rade de Brest. Ainsi, après traitements et hors Guyane (voir section 2), le linéaire commun entre l'indicateur national et Histolitt est long de $7400 \mathrm{~km}$.

\subsection{Rattachement des informations de l'indicateur national et des ouvrages littoraux sur} le tracé de Histolitt

L'indicateur national n'a pas pu être calculé au droit de 6000 profils, dans les cas suivants : (1) au droit des profils recoupant des ouvrages littoraux (qui se substituent au trait de côte), (2) lorsque les photographies aériennes anciennes ne sont pas exploitables, et (3) quand l'environnement a changé (disparition d'une flèche sableuse). Une analyse de la cartographie nationale des ouvrages et aménagements littoraux a permis d'adjoindre aux profils recoupant les ouvrages littoraux (1) le type d'ouvrages (mur, digue, perré, etc.). Les données de l'indicateur national ainsi consolidées sont projetées sur le tracé Histolitt. Cette opération de transfert d'informations entre 2 référentiels différents est rendue possible en découpant le tracé Histolitt en segments longs de 50 mètres au maximum (soit en 210000 segments); puis en rattachant les informations des profils vers les segments les plus proches géographiquement sur l'emprise commune préalablement définie. 


\section{Thème 7 - Risques côtiers}

\section{Analyse des linéaires en évolution}

Les principales évolutions du trait de côte (celles dépassant $0.5 \mathrm{~m} / \mathrm{an}$ ) sont représentées sur la figure 1, sur les $7400 \mathrm{~km}$ du tracé Histolitt.

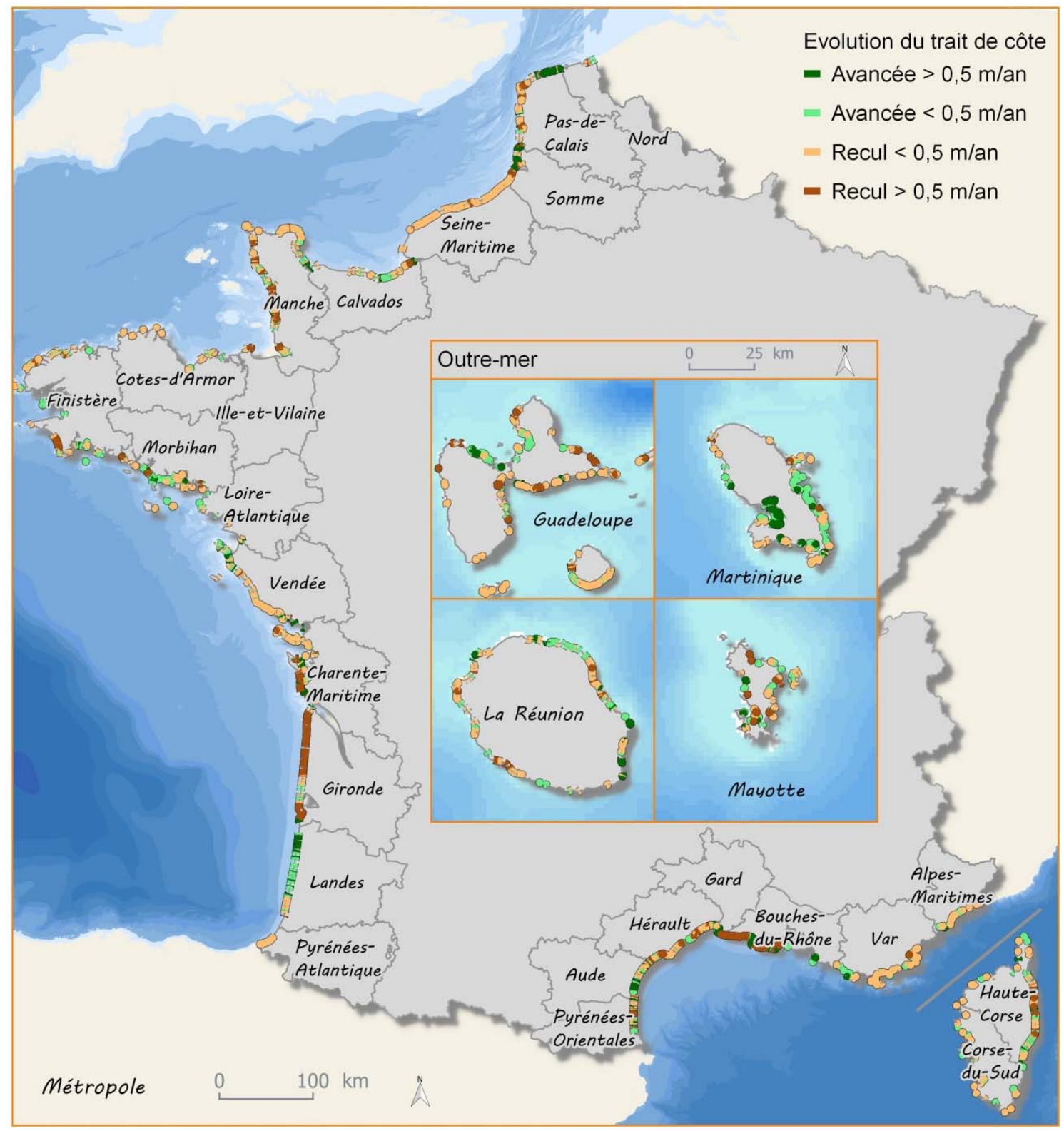

Figure 1. Principales évolutions du trait de côte au niveau national sur la tracé Histolitt (métropole et DROM - hors Guyane).

Sur les territoires étudiés, les reculs les plus importants se concentrent dans les baies des Hauts-de-France, dans les havres du Cotentin, en baie d'Audierne, sur la côte ouest d'Oléron, sur la Côte sauvage, et en estuaire de Gironde, dans le nord du Médoc, autour 


\section{XVİ̀mes Journées Nationales Génie Côtier - Génie Civil \\ Le Havre 2020}

du bassin d'Arcachon et en Camargue (figure 1). En outre-mer, le sud de Grande-Terre en Guadeloupe connaît le plus de recul.

À l'échelle nationale (figure 2), $876 \mathrm{~km}$ de linéaire côtier sont en recul (soit 11,8\% du linéaire), $550 \mathrm{~km}$ sont en avancée (soit 7,4\% du linéaire) et $4353 \mathrm{~km}$ présentent une évolution qualifiée de "non perceptible" (dans un intervalle de $\pm 0,1 \mathrm{~m} / \mathrm{an}$ ) (soit 69,7\% du linéaire). $1244 \mathrm{~km}$ sont fixés par des ouvrages et aménagement littoraux $(16,8 \% \mathrm{du}$ linéaire) et $384 \mathrm{~km}$ n'ont pas pu faire l'objet de calcul d'évolution principalement en raison de photographies anciennes non disponibles (soit 5,2\% du linéaire). Ces résultats n'incluent pas les secteurs portuaires protégés par des jetées, les estuaires, ni les observations effectuées sur le littoral guyanais.

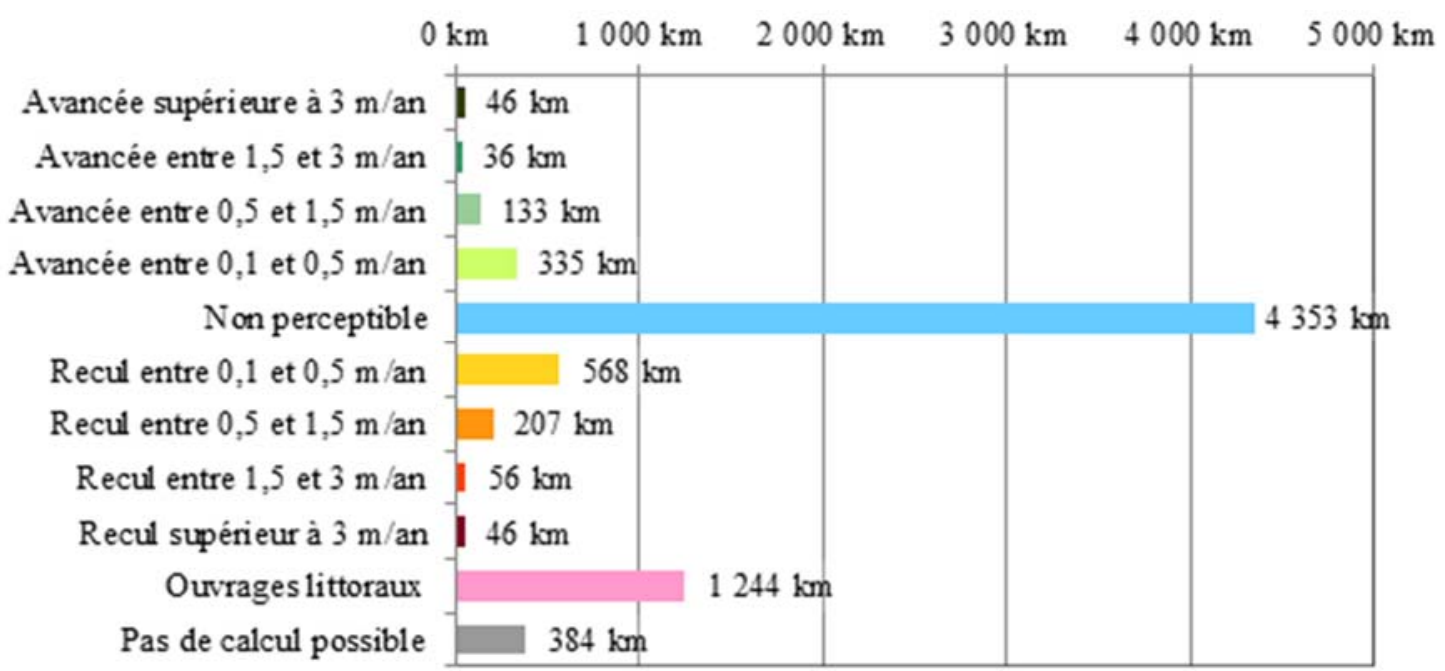

Figure 2. Linéaires (en $\mathrm{km}$ ) des évolutions du trait de côte au niveau national (France métropolitaine et DROM, hors Guyane).

La figure 3 présente l'évolution du trait de côte en fonction de sa géomorphologie. Alors que les falaises et côtes rocheuses représentent près de $4000 \mathrm{~km}$, soit $54 \%$ du linéaire national étudié, seuls environ $160 \mathrm{~km}$ (4\% de ce type de côte) connaissent un recul supérieur à $0,1 \mathrm{~m} / \mathrm{an}$, et concernent principalement les falaises taillées dans des matériaux sédimentaires peu résistants (roches carbonatées : craies, principalement). En revanche, les côtes d'accumulation sableuses représentant $2600 \mathrm{~km}$, soit 35\% du linéaire étudié, sont plus mobiles avec environ $680 \mathrm{~km}$ (26\% du linéaire) en recul, $440 \mathrm{~km}(17 \% \mathrm{du}$ linéaire) en avancée, $810 \mathrm{~km}$ (31\% du linéaire) sans évolution perceptible et $160 \mathrm{~km}(6 \%)$ où l'évolution n'a pas pu être calculée. Sur ces côtes sableuses, $510 \mathrm{~km}$ (soit $20 \% \mathrm{du}$ linéaire) est couvert par la présence d'ouvrages de défense contre la mer. 


\section{Thème 7 - Risques côtiers}

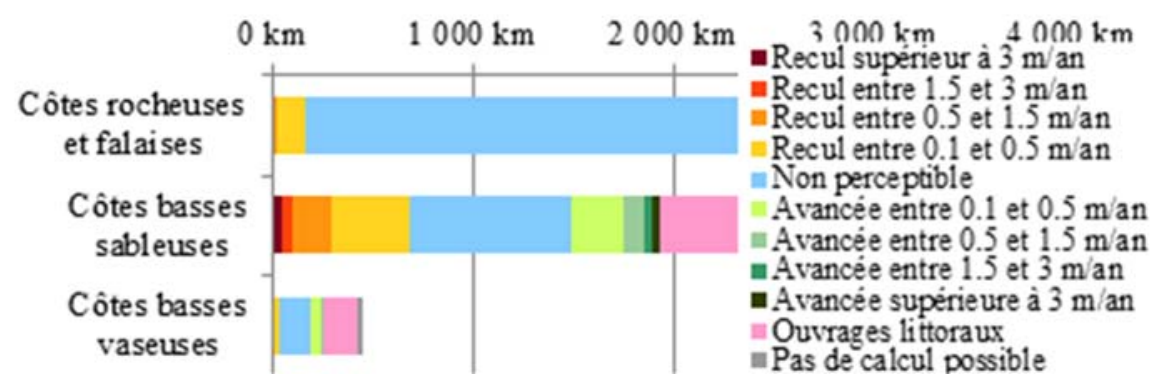

Figure 3. Evolution du trait de côte au niveau national en fonction de la géomorphologie et par linéaire côtier (France métropolitaine et DROM, hors Guyane).

\section{Prise en compte des ouvrages dans les analyses}

Comme mentionné dans la section 4, 16,8\% du linéaire étudiée soit $1244 \mathrm{~km}$, sont fixés par des ouvrages littoraux se substituant au trait de côte. La typologie des ouvrages littoraux, rattachée à Histolitt, permet d'étudier la part d'ouvrages construits spécifiquement pour la protection du littoral (tableau 1). Les ouvrages se substituant au trait de côte pris en compte dans cette analyse sont les "Perré" (364,3 km), les "Mur, mur de soutènement" $(235,2 \mathrm{~km})$ et les "Digue côtière" $(275,7 \mathrm{~km})$ conformément à la typologie de la cartographie nationale des ouvrages et aménagements littoraux (CEREMA \& MTES, 2017).

Tableau 1. Linéaire (en km) de trait de côte en évolution et à ouvrages (France métropolitaine et DROM, hors Guyane).

\begin{tabular}{|c|c|c|c|c|}
\hline \multicolumn{2}{|l|}{ Nature des segments } & Linéaire (km) & $\begin{array}{l}\text { Dont présence } \\
\text { d'épis }\end{array}$ & $\begin{array}{l}\text { Dont présence } \\
\text { de brise-lames }\end{array}$ \\
\hline \multirow{9}{*}{$\begin{array}{l}\text { Evolution du trait de } \\
\text { côte }\end{array}$} & Avancée supérieure à 3 m/an & 46,4 & 1,9 & 0,0 \\
\hline & Avancée entre 1.5 et 3 m/an & 36,2 & 1,0 & 1,2 \\
\hline & Avancée entre 0.5 et $1.5 \mathrm{~m} / \mathrm{an}$ & 133,0 & 7,0 & 3,9 \\
\hline & Avancée entre 0.1 et $0.5 \mathrm{~m} / \mathrm{an}$ & 335,0 & 22,8 & 5,9 \\
\hline & Non perceptible & 4352,8 & 35,1 & 4,3 \\
\hline & Recul entre 0.1 et $0.5 \mathrm{~m} / \mathrm{an}$ & 567,8 & 32,6 & 7,2 \\
\hline & Recul entre 0.5 et $1.5 \mathrm{~m} / \mathrm{an}$ & 207,2 & 23,3 & 3,8 \\
\hline & Recul entre 1.5 et $3 \mathrm{~m} / \mathrm{an}$ & 55,9 & 19,1 & 0,4 \\
\hline & Recul supérieur à 3 m/an & 45,7 & 11,6 & 0,4 \\
\hline \multirow{5}{*}{ Ouvrages } & Brise-lames & 7,0 & 2,9 & 4,0 \\
\hline & Digue côtière & 275,7 & 9,9 & 0,4 \\
\hline & Mur, mur de soutènement & 235,2 & 29,4 & 0,7 \\
\hline & Perré & 364,3 & 60,9 & 1,1 \\
\hline & Autres ouvrages & 362,0 & 20,4 & 4,1 \\
\hline \multirow[t]{2}{*}{$\begin{array}{l}\text { Sans données } \\
\text { d'évolution }\end{array}$} & $\begin{array}{l}\text { Pas de calcul possible de } \\
\text { l'évolution }\end{array}$ & 384,0 & 15,6 & 3,8 \\
\hline & & 7408,2 & 293,7 & 41,1 \\
\hline
\end{tabular}




\section{XVI'mes Journées Nationales Génie Côtier - Génie Civil \\ Le Havre 2020}

Le cas des "brise-lames" est particulier, car positionnés plus vers le large, peu d'entre eux (7 km) ont été rattachés directement sur le tracé Histolitt, d'autres types d'ouvrages situés plus proche du rivage ayant été privilégiés. Un rattachement secondaire est donc réalisé pour les brise-lames. Après traitement, ils sont présents sur $41,1 \mathrm{~km}$ de côte (les très grands brise-lames portuaires de Cherbourg et de Saint-Jean-de-Luz ont néanmoins été exclus du traitement).

La prise en compte des épis, dernier type d'ouvrage de protection, nécessite plus de traitements géomatiques. Afin d'approcher la zone d'influence de ces ouvrages sur le trait de côte, une hypothèse très simplificatrice est retenue : le trait de côte est considéré sous influence d'un épi sur un linéaire de deux fois la longueur de l'épi réparti de part et d'autre de son point central projeté sur le tracé Histolitt. Lorsqu'un épi et un brise-lame sont présents sur le même secteur de côte (cas des épis en "T" par exemple), la présence de l'épi sera privilégiée dans ce rattachement secondaire. Après traitement les épis affectent un linéaire de côte de $293,7 \mathrm{~km}$. Ils concernent $10 \%$ des secteurs en recul et même $30 \%$ des secteurs reculant de plus de 1,5 m/an (tableau 1).

L'addition des linéaires d'ouvrages de protection, suggérant qu'il a eu ou qu'il y a des problèmes d'érosion/submersion, des linéaires concernés par des épis ou des brise-lames (hors secteur déjà recul ou à ouvrages de protection) et des linéaires en recul, correspondant aux zones grisées du tableau 1, montre qu'à minima $1886 \mathrm{~km}$ de trait de côte (876 km initialement sans prise en compte des ouvrages), soit 25,5\% du linéaire total étudié, connaît ou a connu une problématique liée à l'érosion côtière et/ou à la submersion marine. En analysant uniquement les résultats sur les côtes basses sableuses (hors plages de poche), ce pourcentage est de 49\% (1064 km sur $2170 \mathrm{~km})$.

\section{Les limites de la méthode et conclusion}

L'intérêt de ce travail réside également dans l'homogénéité de sa méthode, à savoir, à l'échelle de la France, l'utilisation de mêmes documents d'analyse (photographies aériennes), la définition préalable des traits de côte à observer quel que soit l'environnement littoral rencontré, un même protocole réalisé par le même opérateur. En revanche, ce travail n'est pas dénué de limites liées aux données d'entrée :

a) Comme pour toutes études de photo-interprétation, les limites de l'indicateur national de l'érosion côtière proviennent essentiellement des incertitudes issues du positionnement des traits de côte sur des clichés anciens (années 1950). Par ailleurs, ce type de travaux ne fournit que des vitesses d'évolution entre deux états connus, alors qu'il conviendrait désormais de s'interroger sur les rythmes d'évolution.

b) Les limites de la cartographie nationale des ouvrages et aménagements littoraux sont liées à l'absence de recensement d'ouvrages difficilement visibles par photointerprétation.

c) Les valeurs fournies dans ce travail ont été rapprochées de celles proposées par des études locales récentes issus de travaux universitaires ou d'établissements publics. Des 


\section{Thème 7 - Risques côtiers}

différences ont été observées sur quelques linéaires côtiers très localisés (quelques km) notamment sur des sections côtières très évolutives.

d) Le tracé Histolitt date d'une quinzaine d'année et ne reflète plus toujours la réalité du terrain dans les secteurs très évolutifs. Ce tracé sera prochainement révisé avec la mise en production en 2019 de la "Limite terre-mer" par le Shom et l'IGN.

La méthode de rattachement des informations sur Histolitt peut aussi être questionnée. Le positionnement des données sur des segments longs de 50 mètre (au maximum) engendre une légère incertitude géographique. Cela suppose par ailleurs que les calculs de l'évolution du trait de côte réalisés tous les 200 mètres soient considérés comme représentatifs sur cette distance.

Les limites de cet exercice original à l'échelle nationale sont inévitablement nombreuses, mais les résultats présentés permettent d'accéder aux linéaires en recul en France sur une base de référence connue et largement partagée dans la communauté scientifique. L'ajout des données sur la typologie des ouvrages littoraux complète utilement l'analyse des linéaires soumis aux risques littoraux, notamment sur les secteurs non soumis à évolution perceptible.

\section{Références}

CE -Commission Européenne- (2004). Living with coastal erosion in Europe: sediment and space for sustainability. Office for Official Publications of the European Communities, Luxembourg. Part I "Major findings and Policy Recommendations of the EUROSION project", 10/05/2004, 54 p.; Part II "Maps and statistics", 29/05/2004, 25 p. CEREMA, MTES -Centre d'études et d'expertise sur les risques, l'environnement, la mobilité et l'aménagement \& Ministère de la Transition écologique et solidaire- (2017). Cartographie nationale des ouvrages et aménagements littoraux. http://www.geolittoral.developpement-durable.gouv.fr/cartographie-nationale-des-ouvrages-et-r502.html

CEREMA, MTES -Centre d'études et d'expertise sur les risques, l'environnement, la mobilité et l'aménagement \& Ministère de la Transition écologique et solidaire- (2018). Cartographie de l'indicateur national de l'érosion côtière. http://www.geolittoral.developpementdurable.gouv.fr/indicateur-national-de-l-erosion-cotiere-r473.html

HÉDOU F., ROCHE A., TRMAL C., MORAUD S., DENIAUD Y. (2018). Élaboration de l'indicateur national de l'érosion côtière. XVème Journées Nationales Génie Côtier Génie Civil, La Rochelle, pp. 647-654. https://doi.org/10.5150/jngcgc.2018.075

IGN - Institut Géographique National - SHOM - Service hydrographique et océanographique de la Marine. (2009). Trait de côte Histolitt - v2.0 - Descriptif technique. http://carmenv2.carmencarto.fr/IHM/metadata/MEDITER/Publication/Specifications_techniques_TdCH_v2.pdf MEDTL - Ministère de l'Écologie, du Développement durable, des Transports et du Logement. (2012). Stratégie nationale de gestion intégrée du trait de côte - Programme d'actions 2012-2015. 